PROCEEDINGS OF THE

AMERICAN MATHEMATICAL SOCIETY

Volume 136, Number 6, June 2008, Pages 1925-193

S 0002-9939(08)09310-6

Article electronically published on February 7, 2008

\title{
ALL DIHEDRAL DIVISION ALGEBRAS OF DEGREE FIVE ARE CYCLIC
}

\author{
ELIYAHU MATZRI
}

(Communicated by Martin Lorenz)

\begin{abstract}
In 1982 Rowen and Saltman proved that every division algebra which is split by a dihedral extension of degree $2 n$ of the center, $n$ odd, is in fact cyclic. The proof requires roots of unity of order $n$ in the center. We show that for $n=5$, this assumption can be removed. It then follows that ${ }_{5} \mathrm{Br}(F)$, the 5-torsion part of the Brauer group, is generated by cyclic algebras, generalizing a result of Merkurjev (1983) on the 2 and 3 torsion parts.
\end{abstract}

\section{Mathematical Background}

We begin with basic notions needed for this work and refer the reader to $\underline{8}$ or [5] for more details. Let $R$ be a ring and let $\mathrm{C}(R)=\{r \in R \mid r x=x r \quad \forall x \in R\}$ denote the center of $R$.

Definition 1.1. A ring $R$ will be called a simple ring if $R$ has no non-trivial twosided ideals. In particular $R$ is a division ring if every non-zero element is invertible.

Remark 1.2. Notice that if $R$ is simple, its center is naturally a field.

Definition 1.3. An $F$-algebra $R$ is called an $F$-central simple algebra if $R$ is simple with $\mathrm{C}(R)=F$ and $\operatorname{dim}_{F}(R)<\infty$.

Remark 1.4. Every $F$-central simple algebra $A$ has $\operatorname{dim}_{F}(A)=n^{2}$, and we define the degree of $A$, denoted $\operatorname{deg}(A)$, to be $n$.

By Wedderburn's Theorem every $F$-central simple algebra is of the form $M_{n}(D)$, where $D$ is a division algebra with center $F$.

The Brauer group of a field $F$, denoted $\operatorname{Br}(F)$, is the set of isomorphism classes of $F$-central simple algebras modulo the following relation: two central simple algebras $A, B$ are equivalent if and only if there exist natural numbers $n, m$ such that $M_{n}(A) \cong M_{m}(B)$.

Proposition 1.5. Let $D$ be an $F$-central division algebra of degree $n$, and $K a$ subfield of $D$; then $K$ is a maximal subfield if and only if $[K: F]=n$.

Received by the editors November 27, 2006

2000 Mathematics Subject Classification. Primary 16K20, 12E15.

Key words and phrases. Central simple algebras, cyclic algebras.

The author thanks his supervisors, L. H. Rowen and U. Vishne, for many interesting and motivating talks and for supporting this work through BSF grant no. 2004-083.

(C)2008 American Mathematical Society Reverts to public domain 28 years from publication 
Definition 1.6. A crossed product is an $F$-central simple algebra $A$ of degree $n$ containing a commutative $F$-subalgebra $C$ Galois over $F$, with $[C: F]=n$. Note that if $A$ is a division algebra, then $C$ is a maximal subfield of $A$.

Definition 1.7. Let $D$ be an $F$-central division algebra of degree $n$. We will say that $D$ is split by a group $G$ if $D$ contains a maximal subfield $K$ with Galois closure $E$ such that $\operatorname{Gal}(E / F)=G$.

Theorem 1.8. Let $A$ be a crossed product where $K \subset A$ is a maximal subfield with Galois group $\operatorname{Gal}(K / F)=G$. Then $A$ has the following description: $A=$ $\bigoplus_{\sigma \in G} K x_{\sigma}$ as a left $K$-vector space, and multiplication in $A$ is according to the rules:

$$
x_{\sigma} k=\sigma(k) x_{\sigma} \quad \forall k \in K
$$

and

$$
x_{\sigma} x_{\tau}=c(\sigma, \tau) x_{\tau} x_{\sigma}
$$

where $c \in \mathrm{H}^{2}\left(G, K^{\times}\right)$is a 2-cocycle. In this case $A$ is denoted $A=(K, G, c)$.

Remark 1.9. If $G=\langle\sigma\rangle$ we can give a simpler representation of $A$ as follows: $A=$ $\bigoplus_{i=0}^{n-1} K x^{i}$ as a left $K$-vector space, where $n=\operatorname{deg}(A)=|G|$ and the multiplication is according to the rules

$$
x k=\sigma(k) x \quad \forall k \in K
$$

and

$$
x^{i} x^{j}= \begin{cases}x^{i+j}, & i+j<n \\ \beta x^{i+j-n}, & i+j \geq n .\end{cases}
$$

In this case, $A$ is denoted as $A=(K, \sigma, \beta)$.

Remark 1.10. If $F$ contains a primitive $n$-th root of unity $\rho$, we can give an even simpler description of $A$ (since then $K=F\left[x \mid x^{n}=\alpha \in F\right]$ ) as follows:

$$
A=F\left[x, y \mid x^{n}=\alpha ; y^{n}=\beta ; x y=\rho_{n} y x\right] \quad \alpha, \beta \in F .
$$

\section{Some PRELIMINARY RESUlts}

In this section we briefly repeat the arguments of Rowen and Saltman in 9 but we do not assume $F$ contains roots of unity.

The situation we will be handling is the following: $D / F$ is a central simple algebra of odd degree $n$ having a maximal subfield $K \subset D$ with Galois closure $E \supset K \supset F$, such that

$$
\operatorname{Gal}(E / F)=D_{n}=\left\langle\sigma, \tau: \sigma^{n}=\tau^{2}=1, \tau \sigma \tau=\sigma^{-1}\right\rangle
$$

and $K=E^{\langle\tau\rangle}$.

Extending scalars to $E^{\langle\sigma\rangle}$, we may view $E \subset D^{\prime}=D \otimes E^{\langle\sigma\rangle}$. Now $\operatorname{Gal}\left(E / E^{\langle\sigma\rangle}\right)=$ $\langle\sigma\rangle$, i.e. $D^{\prime}$ is cyclic, so we have an element $\beta \in D^{\prime}$ such that

$$
\beta^{-1} x \beta=\sigma(x) \quad \forall x \in E .
$$

In particular $\beta^{n} \in E^{\langle\sigma\rangle}$. Notice that $\tau$ can be extended to $D^{\prime}=D \otimes E^{\langle\sigma\rangle}$ by its action on $E^{\langle\sigma\rangle}$, that is, we write $\tau$ instead of $1 \otimes \tau$.

Lemma 2.1. We may assume that $\tau(\beta)=\beta^{-1}$. 
Proof. Applying $\tau$ to (1) yields

$$
\tau(\beta)^{-1} \tau(x) \tau(\beta)=\sigma^{-1}(\tau(x)) \quad \forall x \in E .
$$

Now since $\tau$ is an automorphism of $E, \tau(x)$ runs over all elements of $E$, and thus

$$
\tau(\beta)^{-1} y \tau(\beta)=\sigma^{-1}(y) \quad \forall y \in E
$$

that is $\tau(\beta)$ acts on $E$ as $\sigma^{-1}$. Now define $\beta^{\prime}=\beta^{r} \tau(\beta)^{-r}$, where $r=(n+1) / 2$, and compute that $\tau\left(\beta^{\prime}\right)=\beta^{\prime-1}$, and $\beta^{\prime}$ acts on $E$ as $\sigma$.

Let $P_{t}(X)=X^{n}+\sum_{i=1}^{n} c_{i}(t) X^{n-i}$ denote the characteristic polynomial of $t \in$ $D^{\prime}$. Note that $c_{1}(t)=-\operatorname{tr}(t)$ and $c_{n}(t)=(-1)^{n} \mathrm{~N}(t)$ where $\operatorname{tr}(t)$ and $N(t)$ are the reduced trace and norm of $t$.

Lemma 2.2. Let $t=\beta^{i} e$, for $e \in E$ and $0<i<n, i \neq 0$. Then $\operatorname{tr}(t)=0$.

Proof. Let $d=\operatorname{gcd}(i, n)$. Clearly we have $t^{n / d}=\beta^{n i / d} \mathrm{~N}_{\sigma^{i}}(e) \in E^{\left\langle\sigma^{i}\right\rangle}$ where $\mathrm{N}_{\sigma^{i}}$ is the norm from $E$ to $E^{\left\langle\sigma^{i}\right\rangle}$. Now $\left[E: E^{\left\langle\sigma^{i}\right\rangle}\right]=n / d$, implying $P(X)=$ $X^{n / d}-\beta^{n i / d} \mathrm{~N}_{\sigma^{i}}(e)$ is the characteristic polynomial of $t$, hence $\operatorname{tr}_{E / E^{\left\langle\sigma^{i}\right\rangle}}(t)=0$ which implies $\operatorname{tr}_{E / F}(t)=0$.

Lemma 2.3. Let $t=\left(\beta+\beta^{-1}\right)$ e for $e \in E$. Then the coefficients of $P_{t}(X)$ satisfy $c_{i}(t)=0$ for every odd $0<i<n$.

Proof. Notice that for $i$ odd, $t^{i}$ is a sum of elements of the form $a \beta^{s}$ where $a \in E$ and $s$ odd, $-n<s<n$, so by Lemma 2.2 and Newton's identities we are done in the characteristic zero case. For the general case, we refer the reader to 9 ] where the main idea is that you can form a model for this situation in the form of an Azumaya algebra and then use a specialization argument.

Corollary 2.4. There is an element $t \in D$ such that for every $e \in E$ (and so also for $k \in K \subset E), c_{i}=0$ for every odd $0<i<n$ in $P_{t e}(X)$.

Proof. Since $D=D^{\prime\langle\tau\rangle}$ we have $t=\beta+\beta^{-1}$ is the desired element.

Remark 2.5. Notice that if $n=p$ is prime $\operatorname{Char}(F)=p$, the element $t=\beta+\beta^{-1} \in$ $D$ we found satisfies $t^{p} \in F$ and $t \notin F$, and so by a theorem of Albert in the "special results" chapter of his seminal book [1, which is known as Albert's cyclicity criterion, $D$ is cyclic (this is not a new result, as J.P Tignol and P. Mammone did this for any field $F$ with $\operatorname{Char}(F) \mid n$ in [6] using the corestriction, but it shows that the proof of Rowen and Saltman also applies to this case).

\section{THE CASE $n=5$}

Now we would like to focus on the particular case where $n=5$. The main tool we will be using is the following proposition taken from [3, Proposition 2.2].

Proposition 3.1. Let $G\left(x_{1}, \ldots, x_{n}\right)$ be a homogeneous form of degree 3 defined over a field $F$. If $G$ has a solution, $\alpha \in K^{(n)}$, defined over a quadratic extension $K$ of $F$, then $G$ has a solution defined over $F$.

Proof. The proof in [2] uses basic intersection theory which we will not use; instead we will give an algebraic proof (which is actually a translation of the proof in [2]) which will enable us to find an explicit solution in section 3. Since $[K: F]=2$ the solution $\alpha$ has the following form: $\alpha=\left(\alpha_{1}+\beta_{1} t, \ldots, \alpha_{n}+\beta_{n} t\right)$ where $\alpha_{i}, \beta_{i} \in k$, and 
$t \in K$ such that $K=F[t]$. Now specialize $G\left(x_{1}, \ldots, x_{n}\right)$ to $G\left(\alpha_{1}+\beta_{1} Z, \ldots, \alpha_{n}+\beta_{n} Z\right)$, denoting it by $g(Z)$. Notice that the coefficient of $Z^{3}$ in $g(Z)$ is $G\left(\beta_{1}, \ldots, \beta_{n}\right)$; hence if $G\left(\beta_{1}, \ldots, \beta_{n}\right)=0$ we have a solution defined over $F$, otherwise $g(Z)$ is a degree 3 polynomial defined over $F$. Since $g(t)=0$ we get that $g(Z)=c m_{t}(Z)(Z-w)$, where $c=G\left(\beta_{1}, \ldots, \beta_{n}\right)$ and $m_{t}(Z)$ is the minimal polynomial of $t$ over $F$. Now $c, g(Z)$ and $m_{t}(Z)$ are defined over $F$; hence $w$ is in $F$ and clearly $G\left(\alpha_{1}+\beta_{1} w, \ldots, \alpha_{n}+\beta_{n} w\right)=$ $g(w)=0$, so we have found a solution $\gamma=\left(\alpha_{1}+\beta_{1} w, \ldots, \alpha_{n}+\beta_{n} w\right) \in F^{n}$.

Theorem 3.2. Let $D$ be a division algebra of degree 5 split by the group $D_{5}$; then $D$ is cyclic.

Proof. In view of Remark 2.5, we may assume $\operatorname{Char}(F) \neq 5$. First we remark that by Albert's cyclicity criterion it is enough to find an element $t \in D-F$ such that $t^{5} \in F$, that is, $c_{i}=0$ for every $0<i<n$. Now by Corollary 2.4 we have $t \in D$ with the property $c_{i}(t e)=0$ for every odd $0<i<n$ and $\forall e \in E$. Now since $P_{t^{-1}}(x)=-N(t)^{-1} P_{t}\left(x^{-1}\right) x^{5}$ we have $c_{i}\left(e t^{-1}\right)=0$ for every even $0<i<n$ and $\forall e \in E$. Hence we are left with finding a solution for $c_{1}\left(e t^{-1}\right)=0$ (which is linear) and $c_{3}\left(e t^{-1}\right)=0$ (which is cubic) in the five dimensional vector space $E t^{-1}$. Define $V:=\left\{e t^{-1} \in E t^{-1} \mid c_{1}\left(e t^{-1}\right)=0\right\}$, which is a four dimensional subspace of $E t^{-1}$. We have to find a solution for $c_{3}(v)=0$ in $V$. Let us add a fifth root of unity to $F$, which is either a quadratic extension or a chain of two quadratic extensions. After this extension we are in the case of Rowen and Saltman where they gave an explicit element whose fifth power is in $F$ which was $\left(v+v^{-1}\right) t^{-1}$, where $v \in E$. This element is clearly in $V \otimes_{F} F\left[\rho_{5}\right]$. Now by Proposition 3.1 since $c_{3}(v)$ is homogeneous of degree 3 , we have a solution after either one or two quadratic extensions. Thus, we have a solution before the extension, and we are done.

Remark 3.3. If the fifth root of unity is in a quadratic extension of $F$, we know $D$ is cyclic by a theorem of Vishne [10, Theorem 13.6] and D. Haile, M. A. Knus, M. Rost, J. P. Tignol [4, so that what actually is new is the last case of $[F[\rho]: F]=4$.

\section{A generic EXAMPle}

Fixing $p$ let $K=F\left[\rho_{p}\right]$ and denote $\operatorname{Gal}(K / F)=\langle\tau\rangle$. In [7, Theorem 2] Merkurjev proves that ${ }_{p} \operatorname{Br}(F)$ is generated by $F$-central simple algebras, $A$, of degree $p$ such that $A \otimes K \simeq(\alpha, \beta)$ where $K[\sqrt[p]{\alpha}]$ is cyclic over $K$ Galois over $F$.

In [10] Vishne calls these algebras quasi-symbols and gives more details about them, including generic examples. We will show that for $p=5$ these algebras are cyclic and conclude that ${ }_{5} \operatorname{Br}(F)$ is generated by cyclic algebras.

4.1. A generic quasi-symbol of degree 5. For $p=5$ we have two possibilities for $[K: F]$. The first is $[K: F]=2$; in this case Vishne shows that every quasisymbol is cyclic. The second case is $[K: F]=4$; in this case every quasi-symbol $A$ has one of the following forms (after extending scalars to $K$ ):

(1) $A \otimes K=(\alpha, \beta)$, where $\alpha \in F$ and $\tau(\beta) \equiv \beta^{2} \quad\left(\bmod K^{\times^{5}}\right)$.

(2) $A \otimes K=(\alpha, \beta)$, where $\tau(\alpha)=\alpha^{-1}$ and $\tau(\beta) \equiv \beta^{-2} \quad\left(\bmod K^{\times^{5}}\right)$.

The first kind is known to be cyclic by [10, Theorem 10.3]. So we are left with the second kind for which Vishne gives the following generic construction which we will show is cyclic. Thus every quasi-symbol of degree 5 is cyclic and hence, by 7 , Theorem 2] we conclude that ${ }_{5} \operatorname{Br}(F)$ is generated by cyclic algebras. 
Let $k_{0}$ be a field of characteristic $\neq 5$ and $k=k_{0}[\rho]$ where $\rho$ is a fixed primitive fifth root of unity, and $\operatorname{Gal}\left(k / k_{0}\right)=\langle\tau\rangle$ where $\tau(\rho)=\rho^{2}$. Set $K=k(a, b, \eta)$ a transcendental extension and extend $\tau$ to $K$ by

$$
\tau(a)=a^{-1}, \quad \tau(b)=\eta^{5} b^{-2}, \quad \tau(\eta)=\eta^{2} b^{-1} .
$$

Notice that we still have $\tau^{5}=1$. Define $F=K^{\langle\tau\rangle}$ and

$$
D=(a, b)_{K}=K\left[x, y \mid x^{5}=a, \quad y^{5}=b, \quad y x y^{-1}=\rho x\right],
$$

and extend $\tau$ to $D$ by $\tau(x)=x^{-1}, \quad \tau(y)=\eta y^{-2}$. Notice that $\tau^{2}(\eta)=\eta^{-1}$ and $\tau^{2}(y)=y^{-1}$. Now define $D_{0}=D^{\langle\tau\rangle} ; D_{0} / F$ is the generic quasi-symbol of degree 5 of the second type.

Remark 4.1. Vishne's construction is much more general and we specialized it to the above case; for the general construction we refer the reader to [10].

Proposition 4.2. $D_{0}$ is split by $D_{5}$.

Proof. Notice that $\operatorname{Gal}(K[y] / F)=C_{5} \rtimes C_{4}=\langle\sigma\rangle \rtimes\langle\tau\rangle$ and now we will see how $\tau$ acts on $\sigma$. Applying $\tau$ to $x^{-1} t x=\sigma(t)$, which holds for every $t \in K[y]$, yields $\tau(\sigma(t))=\tau\left(x^{-1}\right) \tau(t) \tau(x)=x \tau(t) x^{-1}=\sigma^{-1}(\tau(t))$ and so we get $\tau \sigma \tau^{-1}=\sigma^{-1}$. Hence $\tau^{2}$ is a central element in $\operatorname{Gal}(K[y] / F)$ and it is clear that $E=K[y]^{\left\langle\tau^{2}\right\rangle} \subset$ $K[y]$ is Galois over $F$ with $\operatorname{Gal}(E / F)=D_{5}=\langle\sigma\rangle \rtimes\langle\tau\rangle$, and we are done.

Corollary 4.3. $D_{0}$ is cyclic.

In [7] Merkurjev proves the following theorem:

Theorem 4.4. Let $F$ be a field. ${ }_{n} \operatorname{Br}(F)$ is generated by cyclic algebras, for $n=2,3$.

Now as a result of the above we can extend Merkurjev's theorem to $n=5$ and get

Theorem 4.5. ${ }_{5} \operatorname{Br}(F)$ is generated by cyclic algebras.

Proof. By section 8 of [10] ${ }_{5} \operatorname{Br}(F)$ is generated by quasi-symbols of degree 5 , and so we are done.

4.2. Finding an explicit solution. Since the above example is a generic one, it would be nice to give an explicit element with fifth power in $F$, which is what we do now by going over the general proof.

Let $P_{t}(X)=X^{n}+\sum_{i=1}^{n} c_{i} X^{n-i}$ denote the characteristic polynomial of $t \in$ $D_{0} . \quad V=\left(x+x^{-1}\right)^{-1} K[y]^{\langle\tau\rangle}$ is a 5 -dimensional $F$-subspace of $D_{0}$, satisfying $c_{2}(v)=c_{4}(v)=0$ for all $v \in V$. Also, we want to find a solution in $V$ for $\operatorname{tr}(Z)=$ $c_{1}\left(\left(x+x^{-1}\right)^{-1} Z\right)=0$ and $G(Z)=c_{3}\left(\left(x+x^{-1}\right)^{-1} Z\right)=0$. Extending scalars from $F$ to $F\left[\rho+\rho^{-1}\right]$, we have the solutions $Z_{1}=y+y^{-1}=\alpha+\beta\left(\rho+\rho^{-1}\right)$ and $Z_{2}=\tau\left(Z_{1}\right)=$ $\alpha+\beta \tau\left(\rho+\rho^{-1}\right)=\alpha+\beta \tau\left(\rho^{2}+\rho^{-2}\right)$ where $\alpha=\left(\alpha_{1}, \ldots, \alpha_{5}\right), \beta=\left(\beta_{1}, \ldots, \beta_{5}\right) \in K[y]^{\langle\tau\rangle}$ so $\alpha_{i}, \beta_{i} \in F$. Now define the following line: $L=\{\alpha+\beta t\}=\left\{\left(\alpha_{1}+\beta_{1} t, \ldots, \alpha_{5}+\beta_{5} t\right)\right\}$ defined over $F$.

Proposition 4.6. For every $l \in L$ we have $\operatorname{tr}(l)=0$.

Proof. By standard linear algebra, $L \cap\{\operatorname{tr}(Z)=0\}$ is either one point or the whole line $L$. Since $Z_{1}, Z_{2} \in L \cap\{\operatorname{tr}(Z)=0\}$, we get $L \cap\{\operatorname{tr}(Z)=0\}=L$ and we are done. 
Now let us study the variety $\{G(Z)=0\} \cap L$. First we need to compute $G(Z)$. In order to do that we use the representation of $D$ induced by right multiplication on $D=K[y]+K[y] x+K[y] x^{2}+K[y] x^{3}+K[y] x^{4}$, namely

$$
\begin{gathered}
x \longrightarrow\left(\begin{array}{ccccc}
0 & 0 & 0 & 0 & a \\
1 & 0 & 0 & 0 & 0 \\
0 & 1 & 0 & 0 & 0 \\
0 & 0 & 1 & 0 & 0 \\
0 & 0 & 0 & 1 & 0
\end{array}\right), \\
m \in K[y] \longrightarrow \operatorname{Diag}\left(m, \sigma(m), \sigma^{2}(m), \sigma^{3}(m), \sigma^{4}(m)\right) .
\end{gathered}
$$

Now the minimal polynomial of $x+x^{-1}$ is

$$
\lambda^{5}-5 \lambda^{3}+5 \lambda-\left(a+a^{-1}\right)
$$

hence

$$
\begin{aligned}
\left(x+x^{-1}\right)^{-1} & =\left(\left(x+x^{-1}\right)^{4}-5\left(x+x^{-1}\right)^{2}+5\right)\left(a+a^{-1}\right)^{-1} \\
& =\left(a+a^{-1}\right)^{-1}\left(x^{4}+x^{-4}-x^{2}-x^{-2}+1\right)
\end{aligned}
$$

implying

$$
\left(x+x^{-1}\right)^{-1} \longrightarrow\left(a+a^{-1}\right)^{-1}\left(\begin{array}{ccccc}
1 & a & -1 & -a & 1 \\
a^{-1} & 1 & a & -1 & -a \\
-1 & a^{-1} & 1 & a & -1 \\
-a^{-1} & -1 & a^{-1} & 1 & a \\
1 & -a^{-1} & -1 & a^{-1} & 1
\end{array}\right) .
$$

Now when we compute the characteristic polynomial of $\left(x+x^{-1}\right)^{-1} m$ we get that

$$
\begin{aligned}
c_{3}\left(\left(x+x^{-1}\right)^{-1} m\right)=( & \left.+a^{-1}\right)^{-1}\left(m \sigma(m) \sigma^{2}(m)+\sigma(m) \sigma^{2}(m) \sigma^{3}(m)\right. \\
& \left.+\sigma^{2}(m) \sigma^{3}(m) \sigma^{4}(m)+\sigma^{3}(m) \sigma^{4}(m) m+\sigma^{4}(m) m \sigma(m)\right) \\
= & \left(a+a^{-1}\right)^{-1} \operatorname{tr}_{\sigma}\left(m \sigma(m) \sigma^{2}(m)\right),
\end{aligned}
$$

yielding $F(Z)=\left(a+a^{-1}\right)^{-1} \operatorname{tr}_{\sigma}\left(Z \sigma(Z) \sigma^{2}(Z)\right)$. Now clearly $\{F(Z)=0\} \cap L$ is defined over $F$ by the polynomial

$$
\begin{aligned}
f(t) & \left.=F(\alpha+\beta t)=\left(a+a^{-1}\right)^{-1} \operatorname{tr}_{\sigma}(\alpha+\beta t) \sigma(\alpha+\beta t) \sigma^{2}(\alpha+\beta t)\right) \\
& =\left(a+a^{-1}\right)^{-1} \operatorname{tr}_{\sigma}\left(\beta \sigma(\beta) \sigma^{2}(\beta) t^{3}+\ldots\right)=F(\beta) t^{3}+\ldots .
\end{aligned}
$$

But we know two solutions for $f(t)$, namely $t_{1}=\rho+\rho^{-1}$ and $t_{2}=\rho^{2}+\rho^{-2}$, so we get $f(t)=F(\beta)\left(t-t_{1}\right)\left(t-t_{2}\right)\left(t-t_{3}\right)$. Now since $f(t)$ and $F(\beta)\left(t-t_{1}\right)\left(t-t_{2}\right)$ are defined over $F$, we get $t_{3} \in F$. Explicitly, $f(0)=-t_{1} t_{2} t_{3} F(\beta)$ implies $t_{3}=$ $\frac{-f(0)}{t_{1} t_{2} F(\beta)}=\frac{f(0)}{F(\beta)}=\frac{F(\alpha)}{F(\beta)}$ is in $F$. Hence we get:

Theorem 4.7. The element $w=\left(x+x^{-1}\right)^{-1}\left(\alpha+\beta \frac{F(\alpha)}{F(\beta)}\right) \in D_{0}-F$ satisfies $w^{5} \in F$.

Now we are left with solving for $\alpha, \beta$ from the two equations

$$
\begin{gathered}
y+y^{-1}=\alpha+\beta\left(\rho+\rho^{-1}\right), \\
\eta y^{-2}+\eta^{-1} y^{2}=\tau\left(y+y^{-1}\right)=\alpha+\beta\left(\rho^{2}+\rho^{-2}\right) .
\end{gathered}
$$


Hence

$$
\begin{gathered}
\beta=\frac{y+y^{-1}-\eta y^{-2}-\eta^{-1} y^{2}}{\rho+\rho^{-1}-\rho^{2}-\rho^{-2}}, \\
\alpha=y+y^{-1}-\beta\left(\rho+\rho^{-1}\right) .
\end{gathered}
$$

4.3. The general case. We will now show that the above solution for the case of quasi-symbols, where we descend from $F\left[\rho+\rho^{-1}\right]$ to $F$, is valid for the general case of $D_{5}=\left\langle\sigma, \tau: \sigma^{5}=\tau^{2}=1, \tau \sigma \tau=\sigma^{-1}\right\rangle$ division algebras, where we need to descend from $F[\rho] \otimes E^{\langle\sigma\rangle}$ to $F$. The situation is the following: we look for a solution to $c_{3}(t)=c_{1}(t)=0$ where $c_{i}(t)$ are as in Section 3 and $t \in\left(\beta+\beta^{-1}\right)^{-1} E^{\langle\tau\rangle}$. Let $\operatorname{Gal}(F[\rho] / F)=\langle\pi\rangle$; hence $\operatorname{Gal}(E \otimes F[\rho] / F)=D_{5} \times\langle\pi\rangle$ and so after extending scalars to $F[\rho]$ we want a solution in $\left(\beta+\beta^{-1}\right)^{-1}(E \otimes F[\rho])^{\langle\tau\rangle \times\langle\pi\rangle}$, which will then be defined over $F$.

Proposition 4.8. We may assume $v+v^{-1} \in(E \otimes F[\rho])^{\langle\tau\rangle \times\left\langle\pi^{2}\right\rangle}$, for $v$ as in the proof of Theorem 3.2 .

Proof. Since $v=x^{r} \tau(x)^{-r}$, where $x$ is any eigenvector of $\sigma$ with eigenvalue $\rho$, we may write $x=\sum_{i=0}^{4} \rho^{-i} \sigma^{i}(k)$ for $k \in E^{\langle\tau\rangle \times\langle\pi\rangle}$. Now $\tau(x)=\pi^{2}(x)$ and so $\tau(v)=\tau(x)^{r} x^{-r}=\pi^{2}(x)^{r} x^{-r}=\pi^{2}\left(x^{r} \pi^{2}(x)^{-r}\right)=\pi^{2}\left(x^{r} \tau(x)^{-r}\right)=\pi^{2}(v)$ implying $\tau\left(v+v^{-1}\right)=v+v^{-1}$, hence $v+v^{-1}$ is in $(E \otimes F[\rho])^{\langle\tau\rangle \times\left\langle\pi^{2}\right\rangle}$, as desired.

Now it is clear that after extending scalars to $F\left[\rho+\rho^{-1}\right]$ we have the solution $\left(\beta+\beta^{-1}\right)^{-1}\left(v+v^{-1}\right)$, and so we are in the same situation as in the quasi-symbol case; hence the above solution is valid for the general case, too.

\section{REFERENCES}

1. A.A. Albert, Structure of Algebras, Amer. Math. Soc. Colloq. Publ., Vol. XXIV, 1961. MR0123587 (23:A912)

2. D. F. Coray, Algebraic points on cubic hypersurfaces, Acta Arithmetica 30 (1976), 267-296. MR 0429731 (55:2742)

3. D. Haile, A useful proposition for division algebras of small degree, Proceedings of the American Mathematical Society 106 (1989), 317-319. MR.972232 (89k:16037)

4. D. Haile, M. A. Knus, M. Rost, J. P. Tignol, Algebras of odd degree with involution, trace forms and dihedral extensions, Israel J. Math. 96 B (1996), 299-340 - Amitsur Volume. MR1433693 (98h:16024)

5. N. Jacobson, Finite-Dimensional Division Algebras over Fields, Springer-Verlag, 1996. MR 1439248 (98a:16024)

6. P. Mammone and J. P. Tignol, Dihedral algebras are cyclic, Proceedings of the American Mathematical Society 101 (1987), 217-218. MR902530(89b:12005)

7. A. S. Merkurjev, Brauer groups of fields, Comm. Algebra 11(22) (1983), 2611-2624. MR733345 (85f:12006)

8. L. H. Rowen, Ring theory, Pure and Applied Mathematics, Academic Press, Boston, MA, 1991 (student edition). MR1095047 (94e:16001)

9. L. H. Rowen and David J. Saltman, Dihedral algebras are cyclic, Proceedings of the American Mathematical Society 84 (1982), 162-164. MR637160 (83c:16013)

10. U. Vishne, Galois cohomology of fields without roots of unity, Journal of Algebra 279(2) (2004), 451-492. MR2078127 (2005e:12008)

Department of Mathematics, Bar-Ilan University, Ramat-Gan, 52900, Israel

E-mail address: elimatzri@gmail.com 